\title{
A Feasibility Study to Reduce Infrasound Emissions from Existing Wind Turbine Blades Using a Biomimetic Technique
}

\author{
Jinlei Lv ${ }^{1,2,3}$, Wenxian Yang ${ }^{1, *(\mathbb{C})}$, Haiyang Zhang ${ }^{2,3}$, Daxiong Liao ${ }^{2,3}$, Zebin Ren ${ }^{2,3}$ and Qin Chen ${ }^{2,3}$ \\ 1 School of Engineering, Newcastle University, Newcastle upon Tyne NE1 7RU, UK; 1vjinlei@cardc.cn \\ 2 China Aerodynamics Research and Development Center, Facility Design and Instrumentation Institute, \\ Mianyang 621000, China; zhanghaiyang@cardc.cn (H.Z.); liaodaxiong@cardc.cn (D.L.); qxl@cardc.cn (Z.R.); \\ chenqin@cardc.cn (Q.C.) \\ 3 State Key Laboratory of Aerodynamics, China Aerodynamics Research and Development Center, \\ Mianyang 621000, China \\ * Correspondence: wenxian.yang@ncl.ac.uk; Tel.: +44-191-208-6171
}

Citation: Lv, J.; Yang, W.; Zhang, H.; Liao, D.; Ren, Z.; Chen, Q. A Feasibility Study to Reduce Infrasound Emissions from Existing Wind Turbine Blades Using a Biomimetic Technique. Energies 2021, 14, 4923. https://doi.org/10.3390/ en14164923

Academic Editor: Davide Astolfi

Received: 10 July 2021

Accepted: 6 August 2021

Published: 11 August 2021

Publisher's Note: MDPI stays neutral with regard to jurisdictional claims in published maps and institutional affiliations.

Copyright: () 2021 by the authors. Licensee MDPI, Basel, Switzerland. This article is an open access article distributed under the terms and conditions of the Creative Commons Attribution (CC BY) license (https:// creativecommons.org/licenses/by/ $4.0 /)$.

\begin{abstract}
Infrasound, i.e., low-frequency noise in the frequency range of $10-200 \mathrm{~Hz}$, produced by rotating wind turbine blades has become a matter of concern because it is harmful to human health. Today, with the rapid increase of wind turbine size, this kind of noise is more worrying than ever. Although much effort has been made to design quiet wind turbine blades, today there is still a lack of effective techniques to reduce infrasound emissions from existing blades. To fill this gap in technology, a biomimetic technique that can be readily applied to reduce infrasound emissions of existing wind turbine blades is studied in this paper using both numerical simulation and experimental testing approaches. The numerical study of the technique is based on the analysis of the sound field distribution near the blade, which is derived by performing both aerodynamic and acoustic simulations of the blade. The experimental study of the technique is based on laboratory tests of two scale models of the blade. Both numerical and experimental studies have shown that the shedding vortices behind the blade can be successfully suppressed by semi-cylindrical rings wrapped on the blade. Consequently, both infrasound and the overall sound pressure level of the noise produced by the blade are significantly reduced. Although the rings fail to show good performance in reducing high-frequency noise, it is not a problem for human health because high-frequency noise is weak and moreover it attenuates rapidly as distance increases. The research also showed that the proposed technique can, not only reduce the infrasound produced by the blade, but can also improve the power coefficient of wind turbines.
\end{abstract}

Keywords: biomimetics; wind turbine; blade; infrasound; shedding vortex; power coefficient

\section{Introduction}

In response to the Paris Agreement of 2018, which calls on all countries to take responsibility for tackling climate change and keeping the global temperature rise below 1.5 degrees Celsius in this century, many countries have taken action to accelerate the development of wind power. For example, over $21 \mathrm{GW}$ of wind capacity has been installed in the UK as of 2018 , which contributed $18 \%$ of the electricity generation of the UK in 2018. In the same year, about $41 \%$ of Denmark's electricity demand was supplied by wind power [1]. Moreover, with advances in wind power technology, the size of a single wind turbine is getting larger; however, with the continual growth of wind turbine size, the further development of wind power is facing more challenging issues. These issues are concerning the possible negative impacts of the rapidly growing wind industry on a variety of aspects [2-4]. One of the growing debates is about the potentially harmful effects of wind turbine infrasound on human health.

The noise emitted from a wind turbine is an aggregation of sounds produced by its drive train components (e.g., main bearing, gearbox, generator, etc.) and the rotating 
blades. The noise produced by the drive train components is usually at high frequencies. They are probably annoying to people who work or live near wind farms, but are less concerning; this is because their frequencies are much higher than the natural frequencies of human organs, and their energy attenuates rapidly as distance increases. GE's recent report showed that the noise of a wind turbine will have a sound pressure level (SPL) of $43 \mathrm{~dB}$ at a distance of $300 \mathrm{~m}$ from the turbine, which is close to the SPL of the noise generated by a refrigerator [5]. Thus, high-frequency noises at such SPL levels are not a problem for people who are beyond a distance of $300 \mathrm{~m}$ from the turbine. The worries are mainly focused on the unclear influences of the infrasound produced by the rotating wind turbine blades, which is tonal with a series of frequencies at the blade passing frequency (BPF) and its harmonic frequencies. It was recently reported that the propagation of the sound at the BPF from a $601.6 \mathrm{MW}$ turbine wind farm was detected at a distance of $90 \mathrm{~km} \mathrm{[6].} \mathrm{To} \mathrm{date,} \mathrm{scientists} \mathrm{have} \mathrm{been} \mathrm{working} \mathrm{for} \mathrm{years} \mathrm{to} \mathrm{reveal} \mathrm{the} \mathrm{potential} \mathrm{influences}$ of a blade's infrasound on human health [3-6]. Although there are still many arguments on this topic today, it is wise to take steps to reduce these potential influences of infrasound from rotating blades.

To date, many studies have been conducted to predict the noise of wind turbines. To name a few, Tadamasa and Zangeneh [7] developed codes for calculating the noise radiation of a horizontal axis wind turbine, and their simulation results were verified in an anechoic wind tunnel facility; Luo et al. [8] studied wind turbine aerodynamics and acoustic features by performing numerical simulations and conducting laboratory tests in a wind tunnel. From the research results, some new understandings about the generation and propagation of wind turbine noise were obtained. In $[7,8]$, wind tunnel tests were conducted to verify the simulation results derived from the LES model and the FW-H equation. Ghasemian and Nejat [9] studied the acoustics of vertical-axis wind turbines, focusing on investigating broadband noise in the turbulent boundary layer and the tonal noise at BPF. Although the noise generated by vertical-axis wind turbines is different from that produced by horizontal-axis wind turbines, the methodology developed in the paper is equally applicable to analyzing noises produced by horizontal-axis wind turbines. Apart from the effort to understand the noise emitted from the whole wind turbine system, Lyu et al. [10] proposed a method for reducing the noise emitted from the trailing edge of a wind turbine blade. The experiment showed that an extra sawtooth-edge design was effective to reduce the noise in the vicinity of the trailing edge of the blade. The positive contribution of the sawtooth-edge design to noise reduction was also confirmed by Chen et al. [11]. The sawtooth-edge design changes the flow pattern and reduces the shedding of the airflow from the trailing edge of the blade, thereby reducing the aeroacoustic noise generated by the airflow. In addition to this, an effort was also made to reduce blade noise by optimizing the aerofoil and the structure design of the blade. For example, a multi-objective aerofoil optimization method was proposed by Sanaye [12] to reduce the noise of the aerofoil with the aid of software NAFNoise and XFoil. However, XFoil is not accurate enough when performing calculations that are characterized by a large Reynolds number, thick aerofoil, and a large angle of attack. In addition, due to the noise prediction by NAFNoise is based on the boundary parameter obtained from XFoil, a large noise prediction error is inevitable in the calculation results. For this reason, Wang et al. [13] proposed a new variable to measure the noise radiation of an aerofoil, which was proved to be able to obtain a calculation result that can very well match the testing result. Moreover, the optimized aerofoil obtained by using this method can lead to reduced noise in a specific range of frequencies. In 2020, the author of this paper also carried out similar research and developed an algorithm using SolidWorks, Ansys, and MATLAB in combination to obtain an optimal design of a quieter but more reliable and efficient wind turbine blade [14].

All the aforementioned methods reduce noise by either modifying or optimising the design of the blade and its aerofoils. They are useful for making new quiet blades, but are not applicable to reduce the noise of those wind turbine blades that are already in operation. However, hundreds of thousands of wind turbine blades have already been installed and 
put into operation, and how to reduce the noise, especially infrasound, produced by these existing blades is still an open issue. This motivates the research of this paper. The objective of this study is to develop a feasible post-processing technique that can be easily applied to reduce the infrasound of existing wind turbine blades, while not affecting their energy capture efficiency. Moreover, the proposed technique should not require any modification to the aerofoils and the structure of the blades. The achievement of such a technique will fill the present gap of knowledge in the field of wind power technology and enable us to reduce the infrasound emissions from existing wind turbine blades by using a cost-effective post-processing technique.

The remaining parts of the paper are organized as follows. In Section 2, a new technique for reducing the noise of existing wind turbine blades is proposed. In Section 3, the fundamental theory for noise calculation is briefly reviewed. In Section 4, the effects of the proposed technique on suppressing shedding vortices are numerically and experimentally investigated. In Section 5, the effects of the proposed technique on blade noise and wind turbine power coefficient are investigated. The paper is finally completed in Section 6 with key conclusions and a plan for future research.

\section{A New Technique for Reducing Blade Noise}

Wind turbines produce two kinds of noises in their operation. The first kind is mechanical noise and the second is aeroacoustic noise. The former is made mainly by wind turbine drive train components, such as gearbox, bearing, etc. It is also contributed by holes, slits, and other structural elements of the turbine [15]. This kind of noise is usually characterized by specific frequencies and therefore can be easily identified and reduced using advanced manufacturing and assembly techniques. Aeroacoustic noise has a predominant effect on the environment and human health. It is an aggregation of blade leading-edge noise, boundary layer noise, and blade trailing-edge noise. The leading-edge noise is caused by the interaction of the blade with the stationary airflow in front of the turbine; the boundary layer noise refers to the noise radiated during the transition of the boundary layer near the trailing edge of the blade; the trailing-edge noise is produced by the shedding vortices in the wake area and the blunt trailing edge of the blade [16,17]. To ease understanding, the key regions in a wind turbine blade are illustrated in Figure 1.

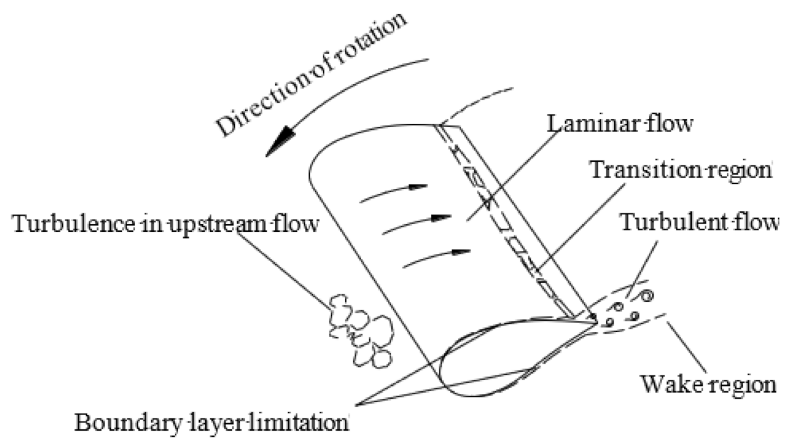

Figure 1. Key regions in a wind turbine blade.

In theory, sound pressure $p$ of the aeroacoustics noise can be estimated by [18]

$$
p \approx \frac{\rho_{0} U_{\infty}^{3} b C_{L} S_{t}}{4 c_{\infty}} \varnothing
$$

where $\rho_{0}$ is the air density; $U_{\infty}$ is the relative speed between airflow and the rotating blade; $b$ refers to the span of the blade; $C_{L}$ is the lift coefficient that is significantly affected by the distribution of the vortices; $S_{t}$ is the Strouhal number; $\varnothing$ denotes a parameter that depends on the distance and direction of noise source; and $c_{\infty}$ represents the sound speed in the free air at infinity. 
Since the distribution of the surrounding airflow can significantly affect the noise radiation of a blade, attempts have been made by scholars to reduce noise by optimising the geometries of the aerofoil to change the surrounding airflow. For example, Maruta et al. [19] developed a low-noise contractive nozzle for performing acoustic tests in a wind tunnel. In their study, semi-cones were installed around the nozzle to break up the vortices, thereby reducing the vortex noise. The author of this paper tried to improve the energy capture efficiency and reduce the noise of a blade by decorating the blade surface using biomimetic features [20]. The research result showed that after applying the biomimetic features to the surface of the blade, the lift-to-drag ratio of the blade was improved while the SPL of the blade noise was reduced at the rated speed of the turbine. Both ref. [19] and ref. [20] suggested that it is possible to reduce the noise and improve the energy capture efficiency of a blade by controlling the surrounding airflow. Encouraged by this finding, a new biomimetic technique is proposed in this paper to reduce the noise of existing wind turbine blades. Considering that blade noise is produced mainly from the tip section of the blade, in this paper, it is proposed to wrap the tip section of the blade using several semi-cylindrical rings (see Figure 2). The reason for only wrapping the tip section of the blade will be further explained in Section 4. In theory, the proposed semi-cylindrical rings will constrain the motion of local airflow on blade surfaces, change the structure of flow boundary, delay and suppress shedding of airflow, and consequently reduce blade noise.

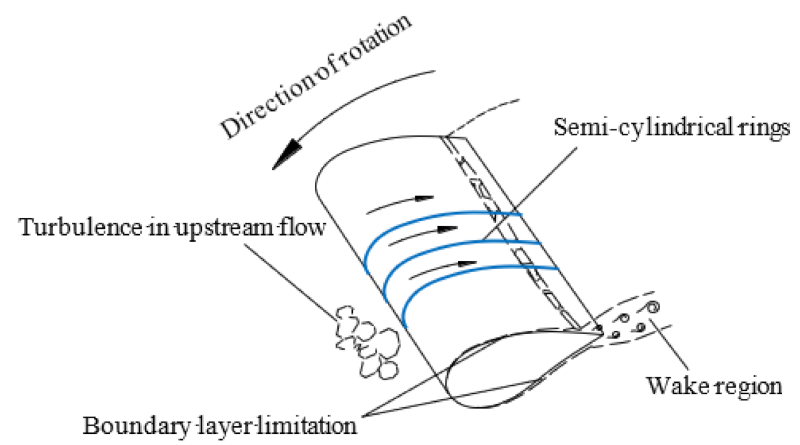

Figure 2. Schematic diagram of the proposed biomimetic technique.

In essence, the proposed technique is a biomimetic technique that is inspired by the leading-edge tubercles on the fin of humpback whales, the trailing-edge profile of the wing of birds, and the strips on the body surface of beetles, as shown in Figure 3. It turns out that they all help to reduce vortex shedding and resistance. In the proposed technique, all these three features are emulated simultaneously using semi-cylindrical rings wrapped on the tip section of wind turbine blades. In other words, the rings wrapped on the blade surfaces can emulate all three kinds of biomimetic features, i.e., "leading edge tubercles", "body strips", and "trailing edge profile", at the same time. Herein, it is worth noting that the three living creatures shown in Figure 3 are just three examples that explain the source of inspiration for the proposed technique. They may not be the best examples to explain the proposed technique, as similar biomimetic features can also be observed from many other organisms. However, they did inspire the research reported in this paper.

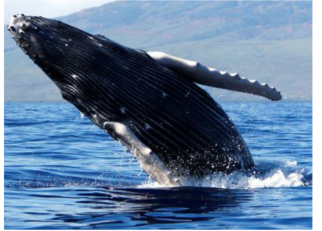

(a)

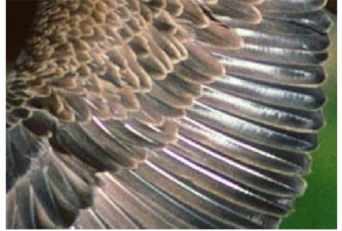

(b)

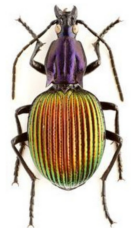

(c)

Figure 3. Sources of inspiration for the proposed technique: (a) leading-edge tubercles on the fin of humpback whale; (b) trailing edge profile of bird wing; (c) strips on the body surface of beetles. 


\section{Fundamental Theory for Calculating Blade Noise}

As done in [21,22], blade noise was predicted in this paper by performing finite element analysis and acoustic analogy calculations. The former was conducted in Ansys Fluent and the latter was implemented in Virtual Lab. Herein, it is worth noting that the large-eddy simulation (LES) model in Ansys Fluent has been widely used to calculate the pressure pulsation on structure surfaces; the aeroacoustics simulation method in Virtual Lab has been popularly used to convert the pressure pulsation data to sound pressure, and then derive sound field propagation from sound pressure. Both software tools have been well proved and popularly used today.

\subsection{The LES Algorithm}

In this study, the LES algorithm was adopted to calculate the pressure pulsation on blade surfaces. As compared to direct numerical simulation (DNS) and Reynolds average Navier-Stokes (RANS) that are used to simulate turbulence in Ansys Fluent, the LES algorithm is more efficient in computation and is, therefore, more favored in practice [7-9]. In the LES algorithm, it is assumed that turbulent flow is composed of many vortices of different scales. Among these vortices, large-scale vortices have more influence on the average flow, turbulent diffusion of various variables, and the exchange of heat, mass, and energy. Moreover, the generation of Reynolds stress is mainly due to those large-scale eddies. By contrast, small-scale vortices mainly affect dissipation. The large-scale vortices are calculated by solving the N-S equation, and the small-scale vortices are calculated based on the sub-grid scale model that describes the relationship between small-scale and large-scale vortices. In the solution of the LES algorithm, the static pressure pulsation is the noise of interest.

In Ansys Fluent, the sub-grid scale turbulent stresses are calculated with the aid of the sub-grid scale turbulence model of the LES based on the Boussinesq hypothesis [23], i.e.,

$$
\tau_{i j}=-2 \mu_{t} S_{i j}+\frac{1}{3} \tau_{k k} \delta_{i j}
$$

where $\mu_{t}$ is the sub-grid scale turbulent viscosity. The isotropic part of the sub-grid scale stresses $\tau_{k k}$ is not modeled but added to the filtered static pressure term. For incompressible flows, this term can be neglected [24]. $S_{i j}$ refers to the rate of strain tensor for the resolved scale. It is expressed as

$$
S_{i j}=\frac{1}{2}\left(\frac{\partial u_{i}}{\partial x_{j}}+\frac{\partial u_{j}}{\partial x_{i}}\right)
$$

The sub-grid scale turbulent viscosity can be obtained from the Smagorinsky-Lilly model, i.e.,

$$
\mu t=\rho L_{s}{ }^{2}|S|
$$

where

$$
|S|=\sqrt{2 S_{i j} S_{j i}}
$$

and $L_{S}$ is the minimum length for sub-grid scales. It is calculated in Ansys Fluent, i.e.,

$$
L_{s}=\min \left(k, d, C_{s}, \Delta\right)
$$

where $k$ is the von Kármán constant, $d$ is the distance to the closest wall, $C_{s}$ is the Smagorinsky constant, and $\Delta$ is the local grid scale that can be computed according to the $1 / 3$ volume of the computational cell.

\subsection{Computation of Aeroacoustics}

Aeroacoustics can be calculated using the following equation, which was proposed in 1969 [25] based on the well-known Lighthill Equation [26]

$$
\frac{1}{a_{0}^{2}} \frac{\partial^{2} p^{\prime}}{\partial t^{2}}-\nabla^{2} p^{\prime}=\frac{\partial}{\partial t}\left\{\left[\rho_{o} v_{n}+\rho\left(u_{n}-v_{n}\right)\right] \delta(f)\right\}-\frac{\partial}{\partial x_{i}}\left\{\left[P_{i j} n_{j}+\rho u_{i}\left(u_{n}-v_{n}\right)\right] \delta(f)\right\}+\frac{\partial^{2}}{\partial x_{i} \partial x_{j}}\left[T_{i j} H(f)\right]
$$


where $a_{0}$ indicates the sound speed in the far-field; $p^{\prime}$ is sound pressure at target position; $f=0$ represents the surface of sound source obtained by integration; $u_{i}$ refers to the velocity component in the direction of $x_{i} ; u_{n}$ and $v_{n}$, respectively, denote the vertical and horizontal direction of speeds towards the surface $f=0 ; \delta(f)$ and $H(f)$ represent Dirac function and Heaviside function, respectively; $P_{i j}$ is stress tensor; and $T_{i j}$ is Lighthill tensor. The three terms on the right side of the equation are associated with the monopole source of thickness noise, the dipole source of loading noise, and the quadrupole source of the noise, respectively.

At present, three methods can be used for calculating aeroacoustics, i.e., computational aeroacoustics (CAA), Lighthill's acoustic analogy method, and a hybrid method that uses the first two methods in combination. Due to the superiorities of the hybrid method to the CAA and Lighthill's acoustic analogy methods in terms of balancing the efficiency and accuracy of noise prediction, it is adopted in this paper to calculate the pressure pulsation in the time domain. Then, the obtained pressure pulsation will be converted to the equivalent sound sources, which are monopole, dipole, or quadrupole sources, or a combinations of them depending on the scenario. Since the hybrid method solves the sound source and sound field separately, it can improve computational efficiency while achieving a more reliable estimation of aeroacoustics.

\section{Effect of the Proposed Technique on Suppressing Shedding Vortices}

Following the brief review of noise calculation theory, the effect of the proposed technique on suppressing the shedding vortices behind the blade will be investigated in this section by both numerical simulation and experimental testing approaches.

\subsection{Numerical Simulation}

The effect of the proposed technique in suppressing shedding vortices will be investigated first using the numerical simulation method. In the calculation, a three-bladed rotor wind turbine $90 / 2500$ produced by FWT Trade GmbH was considered. The rated power of the turbine is $2.5 \mathrm{MW}$ and the diameter of its rotor is $90 \mathrm{~m}$. To facilitate study, a 3D numerical model of the rotor is developed. It is shown in Figure 4a. In the numerical calculation, the wind turbine rotor was placed in a rotating cylindrical computation domain with a diameter of $200 \mathrm{~m}$ and a depth of $60 \mathrm{~m}$, which was meshed by 8 million finite elements. The 3D rotor model was meshed by 4 million finite elements and the meshing results are shown in Figure $4 b$.
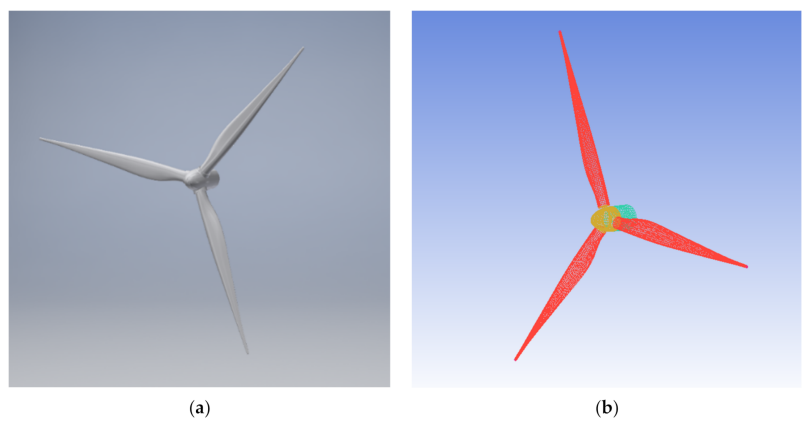

Figure 4. Numerical model of the wind turbine rotor: (a) 3D model of the rotor; (b) meshing results of the rotor.

Firstly, the LES algorithm is used to calculate the steady flow field when the rotor speed is $13 \mathrm{rev} / \mathrm{min}$ (corresponding to a blade tip speed $61.2 \mathrm{~m} / \mathrm{s}$ ). The computational software is Ansys Fluent with Viscous Model Transition SST for 4 equations. The solver is pressure-based, the solution method scheme is coupled, and the numerical model is rigid. Then, the pressure pulsation on the blade surfaces is extracted to identify the noise sources of the blades. The pressure pulsation extraction results are shown in Figure 5. 


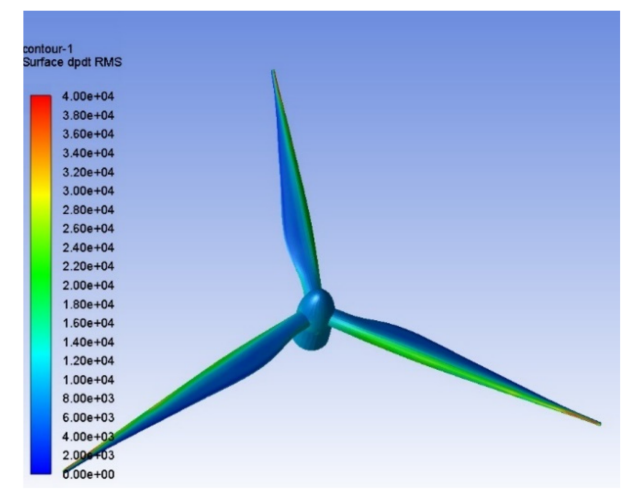

Figure 5. Pressure pulsation on the surfaces of the blades $(\mathrm{kPa})$.

In Figure 5, 'dpdt' refers to an acoustic scale that represents relative noise intensity. From Figure 5, it is found that the most potent zones are located in the tip section of the blades. This calculation result is consistent with published results $[27,28]$. For this reason, the proposed technique will be applied only to the tip section of the blade. Since the linear velocities of the blade in this section are not very different, for the sake of simplicity, a simplified numerical model with a constant aerofoil profile is adopted in the following calculation. The chord length of the aerofoil is $1.5 \mathrm{~m}$. In the calculations, it is assumed that the blade section is wrapped with 17 semi-cylindrical rings, see Figure 6. The diameter of the cross-section of the rings is $D$ and the clearance between adjacent rings is $L$.

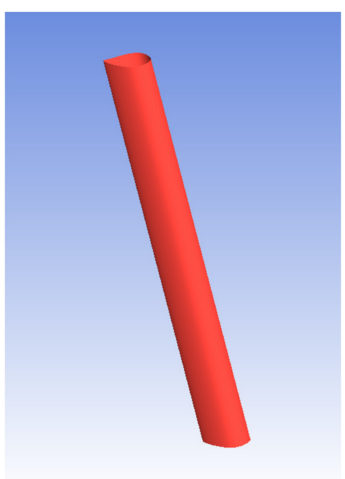

(a)

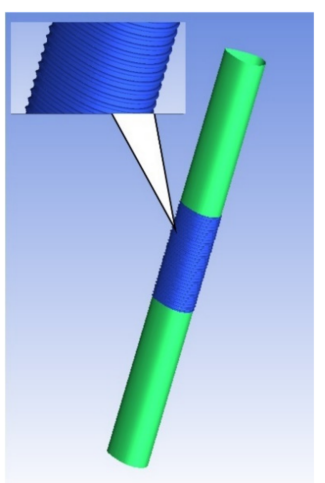

(b)

Figure 6. Numerical models of the tip section of the blade: (a) without rings; (b) with rings.

Then, the transition SST model in Ansys Fluent is adopted to calculate the flow pattern of the airflow around the blade before and after the semi-cylindrical rings are wrapped on the blade. In the calculation, the simplified tip section of the blade was placed in a rectangular computational domain with a length of $14 \mathrm{~m}$, width of $12 \mathrm{~m}$, and depth of $6 \mathrm{~m}$. The computational domain was meshed by 7.8 million finite elements. The inlet of the computational domain is set as "Velocity $60 \mathrm{~m} / \mathrm{s}$ ", the outlet of the domain is "Gauge Pressure $0 \mathrm{~Pa}^{\prime}$. The operating pressure is $101,325 \mathrm{~Pa}$. The $y+$ value near domain walls is about 300 , and the number of iterations is 60,000 . The calculation results, obtained when $D=20 \mathrm{~mm}, L=50 \mathrm{~mm}$ are shown in Figure 7. Where Figure 7a,b shows the streamlines of the airflow obtained before and after using the semi-cylindrical rings, Figure $7 \mathrm{c}$ shows a global view of the streamlines behind the whole blade model. To further show the effect of the proposed technique in suppressing shedding vortices, in Figure $7 \mathrm{c}$, only the middle section of the blade is wrapped with the semi-cylindrical rings, while the other two side sections of the blade are not wrapped with the rings. 


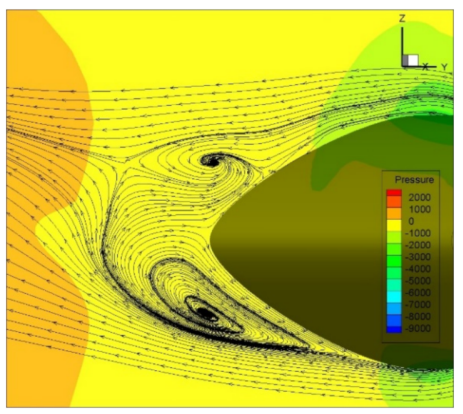

(a)

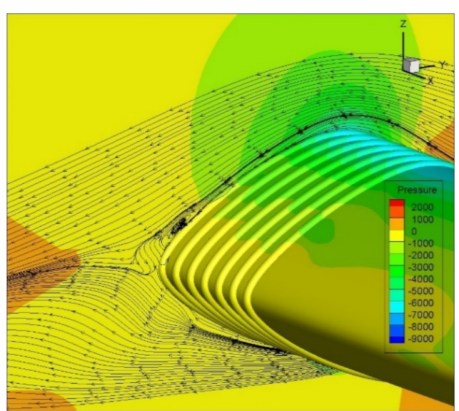

(b)

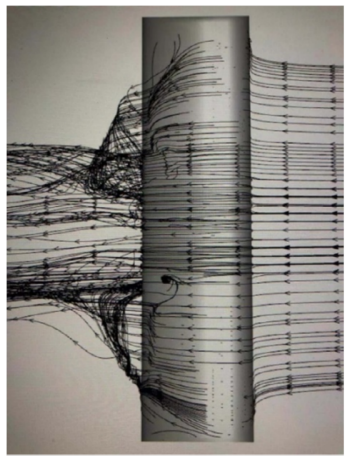

(c)

Figure 7. The pattern of the airflow around the blade: (a) without rings; (b) with rings; (c) global view of the streamlines.

From Figure 7a, it is seen that before using the rings, a pair of anti-rotation shedding vortices are present behind the trailing edge of the blade. They are asymmetric, and significantly disturb the airflow behind the blade. From Figure $7 \mathrm{~b}$, it is interestingly found that after the blade is wrapped with the rings, the shedding vortices that are observed from Figure $7 \mathrm{a}$ are almost completely gone. This suggests that the rings have the potential to suppress the shedding vortices. There is no doubt that the vanishing of the shedding vortices is helpful to improve the efficiency and reduce the noise of the blade. The positive effect of the rings on suppressing shedding vortices can also be observed in Figure 7c. Where the effect of the rings on suppressing shedding vortices can be seen by simply comparing the flow patterns behind the trailing edge of the three parts of the blade. From the figure, it is seen that large shedding vortices are present behind the two side parts of the blade where there are no rings, while the shedding vortices are almost absent behind the trailing edge of the middle part of the blade where the blade is wrapped with rings. Thus, it can be said that the rings wrapped on the blade do work in suppressing shedding vortices.

\subsection{Experimental Tests}

To investigate the actual effect of the semi-cylindrical rings on suppressing shedding vortices in real life, experimental tests were conducted in a laboratory. The scale models used in the tests are shown in Figure 8. Both scale models, coated with tung oil, were made of acrylonitrile butadiene styrene (ABS) and were produced using a 3D printer. 


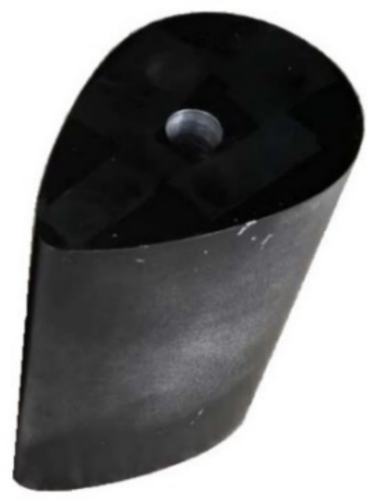

(a)

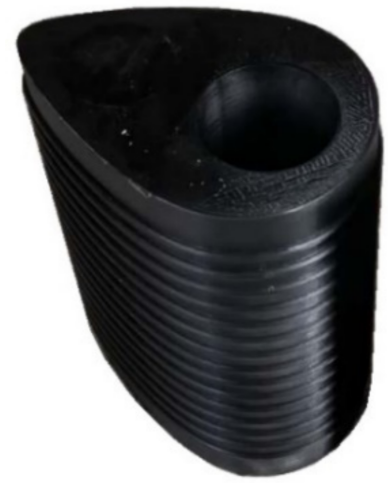

(b)

Figure 8. Scale models used in the tests: (a) without rings; (b) with rings.

The tests were conducted in a $0.55 \mathrm{~m} \times 0.4 \mathrm{~m}$ wind tunnel, see Figure 9. In the tests, the velocity of the airflow was $60 \mathrm{~m} / \mathrm{s}$, and the flow field was measured using high-frequency particle image velocimetry (PIV). The PIV camera is FASTCAM SA-Z, of which the single exposure frequency is $2 \mathrm{kHz}$ and the lens magnification is $3.427 \mathrm{pixel} / \mathrm{mm}$ (the corresponding field of view resolution is $0.3 \mathrm{~mm}$ ). The laser is an Nd:YLF with a single-cavity frequency of $1 \mathrm{kHz}$. The tracer particles are glycerin with a diameter of $1 \mu \mathrm{m}$, which has very good followability at a wind speed of $60 \mathrm{~m} / \mathrm{s}$. The software used for camera data processing is PIVtect Pivviewer2C, and the query window of the camera is 32 pixels $\times 32$ pixels.

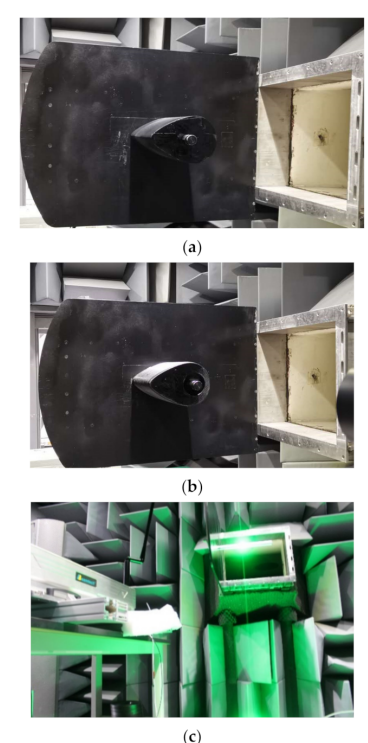



(d)

Figure 9. Experimental tests in wind tunnel: (a) scale model without rings; (b) scale model with rings; (c) test area under laser irradiation; (d) PIV camera and laser.

Then, the streamlines and velocity fields behind the scale models were measured after the wind was applied. The measurement results are shown in Figure 10, where the dark blue areas on the right side of the figures are the trailing edge of the models. Since the laser illuminates from the bottom, the red areas in the figures are the shadow areas of the light path, in which the flow field data are invalid. 


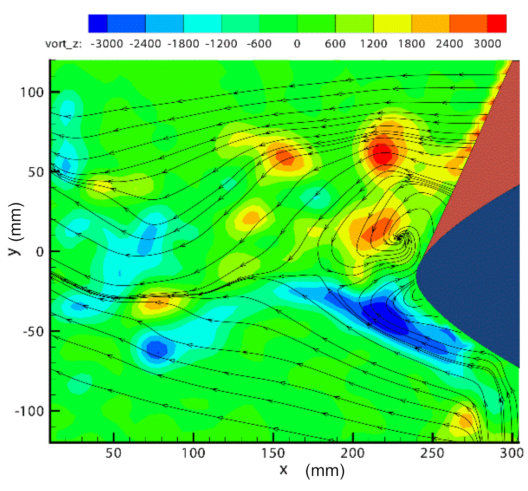

(a)

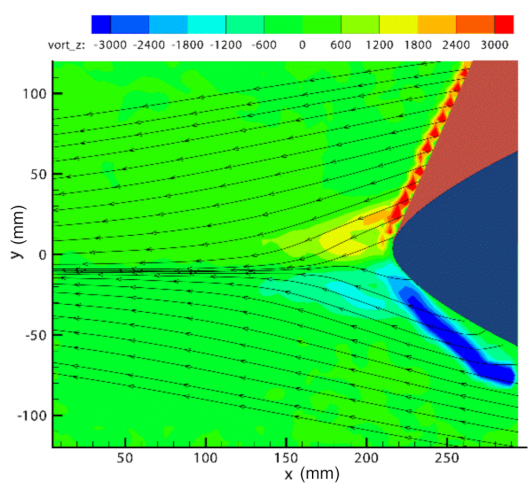

(b)

Figure 10. Velocity fields measured using PIV: (a) scale model without rings; (b) scale model with rings.

From Figure 10, it is seen that shedding vortices appear behind the trailing edge of the blade when the blade is not wrapped with the rings, while the shedding vortices almost disappear when the blade is wrapped with the rings. Such testing results are consistent with the numerical calculation results shown in Figure 7. This fully proves the actual positive contribution of the rings to suppressing the shedding vortices behind the blade in real life. Since it is well known that shedding vortices are one of the major sources of blade noise, it is reasonable to believe that these rings will be effective as well in reducing blade noise. In the meantime, the consistency of the results in Figures 7 and 10 also proves that the numerical models established in Section 4.1 are completely correct, so the calculation results derived from these models are reliable.

\section{Effect of the Proposed Technique on Blade Noise and Wind Turbine Power Coefficient}

\subsection{Effect on Blade Noise}

Following investigating the effectiveness of the proposed technique in suppressing shedding vortices, its effect on reducing the noise emission of the blade is investigated in this section by the approach of acoustics simulation. In the investigation, the time step used in the process of LES calculation is $0.5 \mathrm{~ms}$, which corresponds to a sampling frequency of $2 \mathrm{kHz}$. According to the Nyquist sampling theorem, the frequency range of the noise that can be well predicted in such a calculation will be $0-1000 \mathrm{~Hz}$. To facilitate calculation, the midplane and the centroid-passed horizontal plane of the blade are selected as the acoustic field planes. The remaining boundaries are set as the far acoustic field.

Due to the large value of the Reynolds number in the present case, the turbulent boundary layer will be present over most surfaces of the blade. The turbulent flow will produce noise when it flows over the surface and the trailing edge of the blade. The downstream of the airflow may be separated on the suction side of the aerofoil, thereby generating noise. The vortex shedding noise is also produced by the flow in the shedding region. As opposed to the currently available serrated trailing edge technique that reduces noise by deferring the shedding of the airflow only at the trailing edge of the blade, the proposed technique reduces noise by controlling the airflows not only in the shedding region but also at the leading edge and on the surface of the blade. In other words, the tubercles created by the semi-cylindrical rings on the leading edge can help delay the separation of the airflow from the blade surface. After wrapping 17 semi-cylindrical rings in the tip section of the blade, the radiation maps of noise at different frequencies on the two acoustic field planes are calculated. Herein, it is worth noting that the acoustic calculations in this section are based on the turbulent flow obtained in Section 4.1. In other words, the results of turbulent flow obtained in Ansys Fluent, which are saved in files in the format of "*.cgns", need to be imported into Virtual Lab in advance before starting the acoustic calculation. In the CAA calculation, the number of iterations is 60,000 . The meshes at each 
step, which are saved in files in the format of "*.bdf", are imported from Ansys Fluent as well. The two mutually perpendicular plane fields were meshed by $100 \times 100$ grids. The FEM Standard finite element formulation and the direct solver are selected based on the guideline described in the manual.

To facilitate analysis, when the velocity of the incoming airflow is $60 \mathrm{~m} / \mathrm{s}$, the radiation maps of noise at the frequency of $40 \mathrm{~Hz}$ and $80 \mathrm{~Hz}$ obtained before and after using the rings are shown in Figure 11. Where the intensity of the noise is indicated by greyscales shown in colour bars. In the figures, the red colour represents a larger noise intensity, while the blue colour indicates a smaller noise intensity.

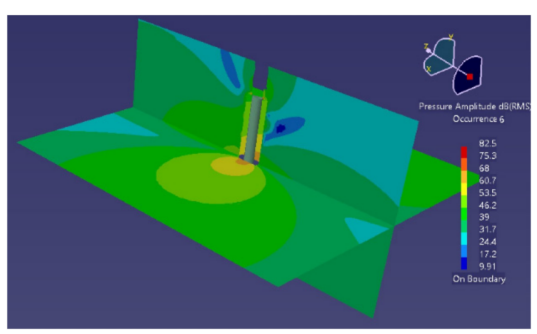

(a)

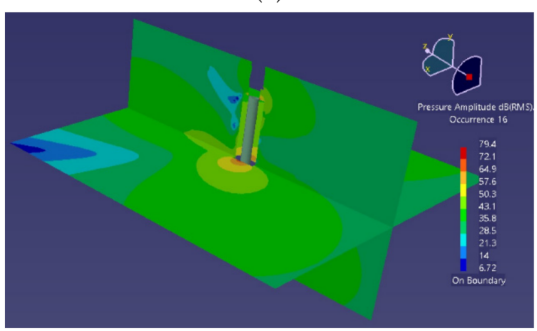

(c)

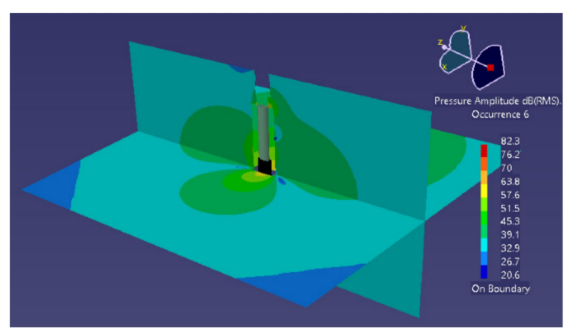

(b)

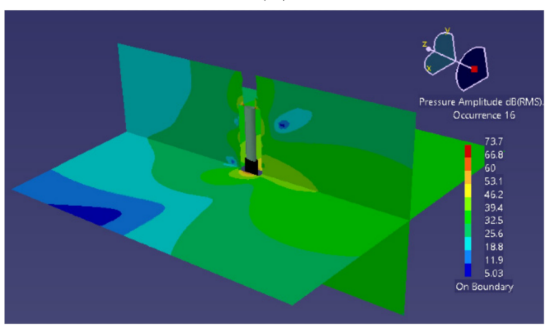

(d)

Figure 11. Radiation maps of noise at different frequencies when flow speed is $60 \mathrm{~m} / \mathrm{s}$ : (a) $f=40 \mathrm{~Hz}$, without rings; (b) $f=40 \mathrm{~Hz}$, with rings; (c) $f=80 \mathrm{~Hz}$, without rings; (d) $f=80 \mathrm{~Hz}$, with rings.

From Figure 11, it is seen that after wrapping 17 semi-cylindrical rings on the blade, the intensities of noise at both frequencies are significantly reduced. This suggests that the rings do reduce the intensity of blade noise. However, the results in Figure 11 only demonstrate the capability of the rings in reducing the noise at frequencies of $40 \mathrm{~Hz}$ and $80 \mathrm{~Hz}$. To investigate the capability of the semi-cylindrical rings in reducing noise in a wider range of frequencies, the broadband noise at a far-field point is calculated before and after using the rings. The far-field point is on the centroid-passed horizontal plane and at a distance of 20 times the chord length away from the blade. The calculation results of the sound pressure at this point are shown in Figure 12. Where the raw sound pressure signals are shown in Figure 12a,b the instantaneous sound pressure level (ISPL) data calculated from the raw sound pressure signals are shown in Figure 12c,d. Herein, it is worth noting that the $\operatorname{ISPL}_{i}(i=1,2, \cdots, n)$ in Figure 12c,d were calculated using

$$
\mathrm{ISPL}_{i}=20 \times \log _{10}\left(\frac{p_{i}}{p_{\text {ref }}}\right) \quad(i=1,2, \cdots n)
$$

where $p_{i}$ refers to the instantaneous sound pressure at the target point; $n=2000$ is the total number of samples; $p_{\text {ref }}=2 \times 10^{-5} \mathrm{~Pa}$ is the reference sound pressure. 


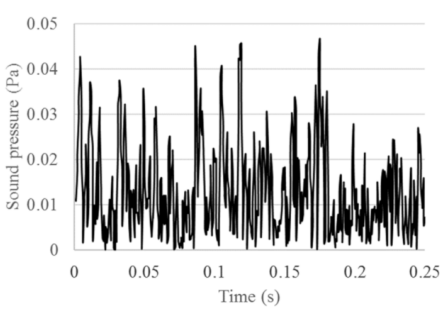

(a)

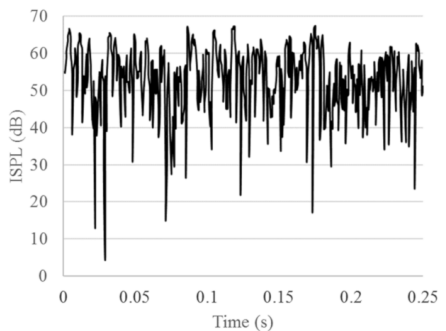

(c)

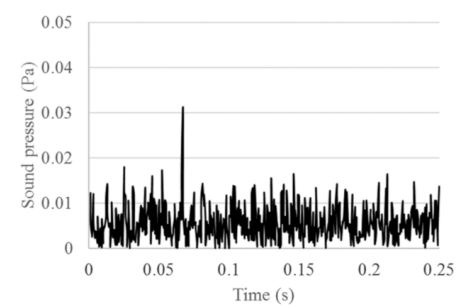

(b)

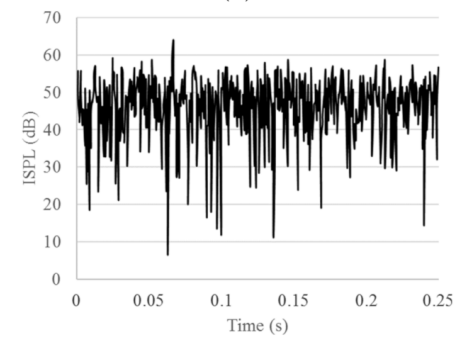

(d)

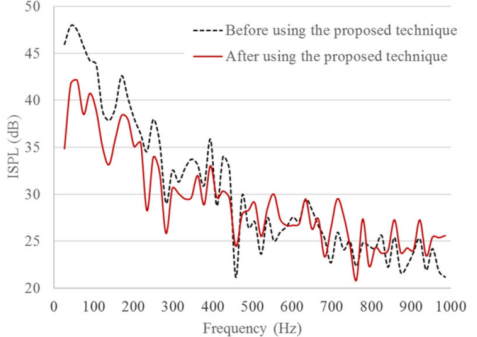

(e)

Figure 12. Broadband noise obtained: (a) sound pressure at the measurement point before using the rings; (b) sound pressure at the measurement point after using the rings; (c) ISPL of the noise before using the rings; (d) ISPL of the noise after using the rings; (e) frequency spectra of the ISPL data obtained in the two scenarios.

In order to show the impact of the proposed technique on the blade noise at a wide range of frequencies, the frequency spectra of the ISPL data are calculated and the calculation results are shown in Figure 12e. Where, the frequency spectra of the ISPL data obtained before and after using the semi-cylindrical rings are plotted together for facilitating comparison.

By comparing Figure $12 \mathrm{a}, \mathrm{b}$ it is clearly seen that after using the rings, the sound pressure at the point of interest has been significantly reduced. This indicates the positive contribution of the rings to the reduction of blade noise. To further investigate the effect of the proposed technique on infrasound, the ISPLs of the sound pressure signals in Figure 12a,b are calculated using (8). Through comparing Figure 12c,d it is found that before using the semi-cylindrical rings, the ISPL of the noise obtained at the measurement point is higher than $60 \mathrm{~dB}$, while it becomes less than $60 \mathrm{~dB}$ after the rings are used. This suggests that the semi-cylindrical rings are indeed helpful in reducing the noise of the blade. Since the public's concern is mainly focused on the infrasound, in the frequency range of $10-200 \mathrm{~Hz}$, generated by wind turbine blades, the frequency spectra of the noise are also investigated in order to understand the capability of the proposed technique in reducing the infrasound made by the blade. From Figure 12e, it is found that the proposed technique is particularly effective in reducing the infrasound in the frequency range of 10 200 Hz. Since the noise in this frequency band is harmful to human health, it can be said that the proposed technique makes wind turbines more friendly to human health. Herein, it is worth noting that although the proposed technique does not very well suppress the blade's high-frequency noises (e.g., $>400 \mathrm{~Hz}$ ), this is not a problem. That is because, on the one hand, these high-frequency noises are weak and on the other hand, they will attenuate rapidly as distance increases. So, they can cause little annoyance. 
To evaluate the general effect of the proposed technique on noise reduction, the frequency domain overall sound pressure levels (OASPLs) of the sound pressure signals in Figure 12 are evaluated using the following equation

$$
\mathrm{OASPL}=10 \times \log _{10}\left(\sum_{k=1}^{m} 10^{\frac{\mathrm{ISPL}_{k}}{10}}\right)
$$

where $m$ is the total number of frequency components, ISPL $L_{k}$ indicates the instantaneous sound pressure level of the $i$-th frequency component of the noise.

With the aid of (9), it can be readily known that the frequency domain OASPL obtained before using the rings is $55.5 \mathrm{~dB}$, while this value drops to $50.9 \mathrm{~dB}$ after using the rings. In other words, the frequency domain OASPL of the noise is reduced by $8.3 \%$ (i.e., $4.6 \mathrm{~dB}$ ) by the rings.

\subsection{Effect on Wind Turbine Power Coefficient}

The effect of the proposed technique on the energy capture efficiency of the wind turbine is further investigated in this section by calculating its power coefficient $C_{P}$ before and after its blades are wrapped with semi-cylindrical rings. Considering the rings are wrapped only on the tip section of the blades where the noise radiation intensity is high, only the aerodynamic performance of this part of the blade is investigated in the following. Due to the limited computing resource, only three rings are wrapped on the tip section of the blades in the calculation. The corresponding numerical model of the tip section of the blade and its meshing results are shown in Figure 13. Herein, the tip section of the blade refers to 44-48 $\mathrm{m}$ from the root of the blade, and the chord length of the aerofoil changes from $1.5 \mathrm{~m}$ to $1.95 \mathrm{~m}$.

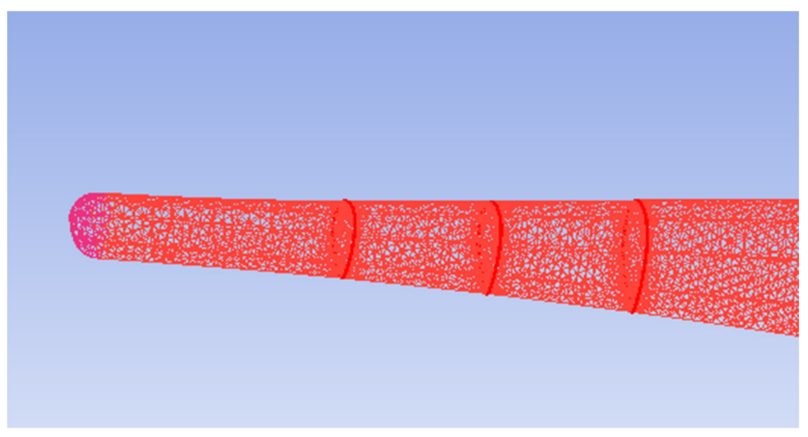

Figure 13. Meshing result of the tip section of the blade.

It is well known that the power coefficient $C_{P}$ of the wind turbine is a function of the tip speed ratio $R_{t}$ of the blade, which is the ratio between the tangential speed $V$ of the blade tip and the speed $v$ of the incoming airflow. The power coefficient $C_{P}$ can be calculated by

$$
C_{P}=\frac{T \omega}{\frac{1}{2} \rho A v^{3}}=\frac{2 T V}{\rho r A v^{3}}=\frac{2 T}{\rho r A v^{2}} \times R_{t}=\frac{T}{r A q} \times R_{t}
$$

where $T$ indicates the torque of the rotor, $\omega=\frac{V}{r}$ is the angular speed of the rotor, $r$ is the radius of the rotor, $\rho$ represents the air density. $A$ is the swept area of the rotor, $R_{t}=\frac{V}{v}$ is tip speed ratio of the blade, and $q=\frac{1}{2} \rho v^{2}$ is the dynamic pressure of free airflow.

By using (10), the power coefficient $C_{P}$ is calculated when the tip speed ratio $R_{t}=5,8,11$, and 14.5, respectively. The corresponding calculation results obtained at these tip speed ratios before and after using the rings are shown in Figure 14. 


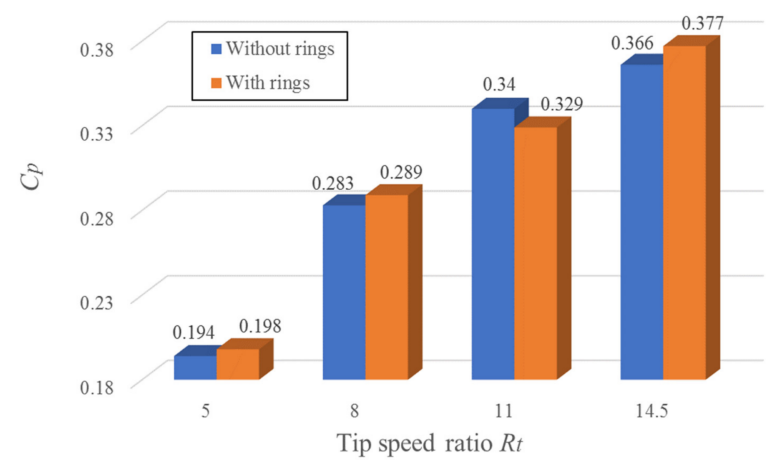

Figure 14. The $C_{P}$ obtained at different tip speed ratios.

From Figure 14, it is seen that after wrapping three rings on the tip section of the blades, the power coefficient $C_{p}$ of the wind turbine increases at all tip speed ratios except for 11 . The reason why the power coefficient value becomes lower when the tip speed ratio is 11 is currently unclear, because many factors can affect the numerical calculation results. Herein, it is worth noting that large scale three-bladed wind turbines usually operate at a tip speed ratio of 6 to 8 , with 7 being the most widely reported value. Therefore, the results in Figure 14 suggest that the proposed technique not only has a great potential to reduce the infrasound emissions from the blades but also has the potential to improve the energy capture efficiency of the wind turbine. Such a conclusion can also be inferred from the results given in Section 5.1, in which it was reported that the frequency domain OASPL of the noise is reduced by $8.3 \%$ after using the rings. According to the conservation law of energy, that means after using the rings, less energy is converted to be noise and more energy is captured by the blades. Therefore, the power coefficient of the wind turbine is improved accordingly.

\section{Conclusions}

With the increasing application of wind turbines, the infrasound produced by rotating wind turbine blades has become a matter of concern because they are harmful to human health. To achieve quiet wind turbine blades, a great deal of effort has been made. However, all achieved techniques are designed for making new quiet blades, and none of them can be used to reduce infrasound emissions from an existing blade. To fill this gap in technology, a biomimetic technique is proposed in this paper. From the research reported above, it can be concluded that:

- As opposed to the currently available techniques that reduce noise by optimising the aerofoil or the structural design of wind turbine blades, the proposed technique is easier to be used to reduce the infrasound produced by existing wind turbine blades as it does not need to make any change to the aerofoils and blade structures;

- The proposed technique is cost-effective and easy to use on existing wind turbine blades as it only needs to wrap several semi-cylindrical rings on the tip section of the blades;

- $\quad$ The proposed technique does have the potential to reduce the infrasound produced by rotating wind turbine blades;

- The proposed technique can, not only reduce infrasound, but can also improve the power coefficient of the wind turbine. Since the tip speed ratio of most commercial three-bladed wind turbines is between 6 and 8, the proposed technique can fully meet the needs of commercial wind turbines for post-improvement in noise and efficiency.

Despite the encouraging results reported above, a more in-depth study of the proposed technique, including the influences of ring material, the geometric shape, size, and the number of rings, as well as the clearance between them on blade infrasound, will be conducted in the future to further improve the effectiveness of the proposed technique. In addition, it is worth noting that the noise inside the wind tunnel before and after using 
the rings was not measured in the reported experiment. This is because the PIV camera and laser device for measuring shedding vortices were installed in the wind tunnel in the experiment. They occupied a large part of the space in the wind tunnel (see Figure 9d) and may significantly affect the accuracy of noise measurement, thereby making it impossible to use the measured noise to verify the actual effect of the proposed technique. When the wind tunnel is available in the future, all equipment in the wind tunnel will be removed and noise measurements will be conducted in the wind tunnel. The relevant research will be reported in a separate paper.

Author Contributions: Methodology, investigation, writing-original draft preparation, J.L.; Conceptualization, methodology, investigation, supervision, project administration, writing-review and editing, W.Y.; software, H.Z. and D.L.; funding acquisition, resources, Z.R. and Q.C. All authors have read and agreed to the published version of the manuscript.

Funding: This research received no external funding.

Institutional Review Board Statement: Not applicable.

Informed Consent Statement: Not applicable.

Data Availability Statement: Data sharing is not applicable to this article.

Conflicts of Interest: The authors declare no conflict of interest.

\section{References}

1. Wind Europe. Wind Energy in Europe in 2018, Trends and Statistics, February 2019. Available online: https://windeurope.org/ wp-content/uploads/files/about-wind/statistics/WindEurope-Annual-Statistics-2018.pdf (accessed on 8 July 2021).

2. Abbasi, S.A.; Abbasi, T.; Abbasi, T. Impact of wind-energy generation on climate: A rising spectre. Renew. Sustain. Energy Rev. 2016, 59, 1591-1598. [CrossRef]

3. Carlile, S.; Davy, J.L.; Hillman, D.; Burgemeister, K. A review of the possible perceptual and physiological effects of wind turbine noise. Trends Hear. 2018, 22, 1-10. [CrossRef] [PubMed]

4. Jakobsen, J. Infrasound emission from wind turbines. J. Low Freq. Noise Vib. Act. Control 2005, 24, 145-155. [CrossRef]

5. Kellner, T. How Loud Is a Wind Turbine? GE Reports, 2 August 2014. Available online: https://www.ge.com/news/reports/ how-loud-is-a-wind-turbine (accessed on 8 July 2021).

6. Marcillo, O.; Arrowsmith, S.; Blom, P.; Jones, K. On infrasound generated by wind farms and its propagation in low-altitude tropospheric waveguides. J. Geophys. Res. Atmos. 2015, 120, 9855-9868. [CrossRef]

7. Tadamasa, A.; Zangeneh, M. Numerical prediction of wind turbine noise. Renew. Energy 2011, 36, 1902-1912. [CrossRef]

8. Luo, K.; Zhang, S.; Gao, Z.; Wang, J.; Zhang, L.; Yuan, R.; Fan, J.; Cen, K. Large-eddy simulation and wind-tunnel measurement of aerodynamics and aero-acoustics of a horizontal-axis wind turbine. Renew. Energy 2015, 77, 351-362. [CrossRef]

9. Ghasemian, M.; Nejat, A. Aero-acoustics prediction of a vertical axis wind turbine using Large Eddy Simulation and acoustic analogy. Energy 2015, 88, 711-717. [CrossRef]

10. Lyu, B.; Azarpeyvand, M.; Sinayoko, S. Prediction of noise from serrated trailing edges. J. Fluid Mech. 2016, 793, 556-588. [CrossRef]

11. Chen, E.; Ma, Y.; Yang, A.; Zhao, G. Experimental investigation on noise emissions of an airfoil with non-flat plate trailing edge serrations. J. Mech. Sci. Technol. 2019, 33, 3069-3074. [CrossRef]

12. Sanaye, S.; Hassanzadeh, A. Multi-objective optimization of airfoil shape for efficiency improvement and noise reduction in small wind turbines. Renew. Sustain. Energy 2014, 6, 053105. [CrossRef]

13. Wang, Q.; Wang, X.; Yang, J.; Wang, J.; Sun, J.; Qin, Z. Aerodynamic optimal design of low noise wind turbine blades. China Mech. Eng. 2018, 29, 1574-1579, 1587.

14. Li, Y.; Wei, K.; Yang, W.; Wang, Q. Improving wind turbine blade based on multi-objective particle swarm optimization. Renew. Energy 2020, 161, 525-542. [CrossRef]

15. Tonin, R. Sources of wind turbine noise and sound propagation. Acoust. Aust. 2012, 40, 20-27. Available online: https: / / docs.wind-watch.org/Tonin-wind-turbine-noise-and-propagation.pdf (accessed on 8 July 2021).

16. Yang, B. Research Status on Aero-Acoustic Noise from Wind Turbine Blades. IOP Conf. Ser. Mater. Sci. Eng. 2013, 52, 012009. Available online: https://iopscience.iop.org/article/10.1088/1757-899X/52/1/012009/pdf (accessed on 8 July 2021). [CrossRef]

17. Brooks, T.F.; Pope, D.S.; Marcolini, M.A. Airfoil Self-Noise and Prediction. NASA Reference Publication 1218, July 1989. Available online: https:/ /ntrs.nasa.gov/api/citations/19890016302/downloads/19890016302.pdf (accessed on 8 July 2021).

18. Glegg, S.; Devenport, W. Aeroacoustics of Low Mach Number Flows: Fundamentals, Analysis and Measurement; Academic Press: Cambridge, MA, USA, 2017.

19. Maruta, Y.; Minorikawa, G.; Maruta, Y.; Minorikawa, G. Developments of low-noise contractive nozzle used in acoustic windtunnel. In Proceedings of the 3rd AIAA/CEAS Aeroacoustics Conference, Atlanta, GA, USA, 12-14 May 1997. 
20. Yang, W.; Alexandridis, T.; Tian, W. Numerical research of the effect of surface biomimetic features on the efficiency of tidal turbine blades. Energies 2018, 11, 1014. [CrossRef]

21. Reese, H.; Carolus, T. Axial fan noise: Towards sound prediction based on numerical unsteady flow data-A case study. J. Acoust. Soc. Am. 2008, 123, 3539. [CrossRef]

22. Schram, C.; Bériot, H.; Roger, M.; Reese, H.; Carolus, T. On the importance of near-field terms in the assessment of acoustic installation effects for ducted low-Mach number fans. In Proceedings of the 15th AIAA/CEAS Aeroacoustics Conference (30th AIAA Aeroacoustics Conference), Miami, FL, USA, 11-13 May 2009.

23. Hinze, J.O. Turbulence; McGraw-Hill Publishing, Co.: New York, NY, USA, 1975.

24. Erlebacher, G.; Hussaini, M.Y.; Speziale, C.G.; Zang, T.A. Toward the large-eddy simulation of compressible turbulent flows. J. Fluid Mech. 1992, 238, 155-185. [CrossRef]

25. Ffowcs Williams, J.E.; Hawkings, D.L. Sound generation by turbulence and surfaces in arbitrary motion. Philos. Trans. R. Soc. A Math. Phys. Eng. Sci. 1969, 264, 321-342. [CrossRef]

26. Lighthill, M.J. On sound generated aerodynamically. I. General theory. Proc. R. Soc. A Math. Phys. Eng. Sci. 1952, $211,564-587$. [CrossRef]

27. Norsonic Acoustic Camera-Wind Turbines Noise.mov. Available online: https://www.youtube.com/watch?v=6lxt9KeEoSA (accessed on 8 July 2021).

28. Wind Turbine Blade Noise. Available online: https:/ / www.youtube.com/watch?v=o225pybRUGs (accessed on 8 July 2021). 